\title{
Auditory delayed discriminations by the dolphin: Nonequivalence with delayed-matching performance
}

\author{
ROGER K. R. THOMPSON \\ Franklin and Marshall College, Lancaster, Pennsylvania I7604 \\ and \\ LOUIS M. HERMAN \\ University of Hawaii, Honolulu, Hawaii 96822
}

\begin{abstract}
Working memory in a bottlenosed dolphin was tested in both indirect and direct auditory delayed-discrimination tasks in which a correct spatial response was conditional upon the nature of a preceding sound. In the indirect task, either one of two possible sounds was briefly presented. After a prescribed delay, the dolphin was cued to go either to a left-hand or right-hand paddle pair. Responses to the outer paddle of a pair were rewarded following sound $A$, and responses to the inner paddle of a pair were rewarded after sound $B$. In the direct delayeddiscrimination task, only one paddle pair was used in each session. In both tasks, the delay interval between the discriminative sound stimulus and the opportunity for a spatial response was progressively increased over sessions until the animal failed to meet a specified performance criterion or self-terminated a session. Delay limits of about 30 and $60 \mathrm{sec}$ were obtained in the indirect and direct tasks, respectively. The increase in delay limit in the latter task was attributable to the use of an overt mediational response during the longer delays. In both cases, however, the obtained delay limits fell considerably short of the 2- to 3-min limits obtained in auditory delayed-matching studies using the same test sounds and the same subject. The task differences indicate that working memory functions cannot depend upon memory of the predelay stimulus alone, but must be determined in part by additional processes.
\end{abstract}

Recent studies using delayed matching-to-sample procedures have shown that the bottlenosed dolphin's (Tursiops truncatus) working memory for sound is excellent. It easily remembers either single novel or familiar sounds over delays of 2 min or more and can recognize elements from lists of five or six sounds (Herman, 1975; Herman \& Gordon, 1974; Thompson \& Herman, 1977). These findings are in keeping with what is known of this species' well-developed peripheral and central auditory nervous system (Kruger, 1966). In contrast, the dolphin's memory for visual form or brightness stimuli is quite poor (Herman, 1980). The modality differences in memory skills hint at some of the specializations and constraints in dolphin memory, an issue taken up in greater detail elsewhere (Herman, in press).

This paper is based on a portion of a dissertation submitted by the first author in partial fulfillment of the requirements of the PhD degree at the University of Hawaii. This research was supported by Grant GB-32148X from the National Science Foundation to the second author and is Contribution No. 592 from the Hawaii Institute of Marine Biology. Reprint requests should be sent to Roger K. R. Thompson, Whitely Psychology Labs, Franklin and Marshall College, P.O. Box 3003, Lancaster, Pennsylvania 17604.
The importance of retrieval processes in animal memory has been emphasized by a number of workers (e.g., Spear, 1971, 1978), and the postdelay comparison choice stimuli used in delayed-matching procedures can be thought of as retrieval cues for predelay sample stimulus traces (D'Amato \& Worsham, 1974). Herman and Thompson (Note 1), using delayedmatching procedures, studied the effects of direct (unconditional), conditional, and probe-type auditory retrieval cues on the dolphin's memory for familiar sounds. In the conventional delayed-matching task used to test the effects of direct retrieval cues, the dolphin first heard one of two highly familiar sample sounds, A or B. After a delay (the retention interval), two comparison sounds, identical to the possible sample sounds, were presented. The dolphin's memory was tested by requiring her to recognize the comparison sound matching the sample on that particular trial. In the delayed-probe-matching condition, only a single retrieval cue (i.e., the probe) was presented at the end of the delay following the presentation of either sound A or B as sample. The dolphin was rewarded for responding to the probe if it was identical to the sample and for responding to an alternate silent speaker if the probe and sample dif- 
fered. In the conditional procedure, two retrieval cues were presented, as in the conventional delayedmatching task, but they differed in quality from the sample sounds with which they were respectively associated. For example, following sample-sound A, choosing comparison-sound $\mathrm{C}$ was correct, whereas following sample-sound $\mathrm{B}$, comparison-sound $\mathrm{D}$ was the correct choice.

The results of the Herman and Thompson (Note 1) study indicated a near equivalence of performance across the three conditions and also were consistent with theoretical interpretations of delayed-matching performances as reflecting memory for the relative recency of sounds as sample (D'Amato, 1973). In each case, the dolphin's performance level was equal to or better than $90 \%$ correct through delays of $50 \mathrm{sec}$ and, beyond that point, never fell below $80 \%$ correct responses through at least a 90 -sec delay.

Memory for the sample sounds obviously remained robust despite the described variations in the available retrieval information and its relationship to the prior sample sounds. These results suggest that the different postdelay comparison sounds had no differential retrieval function but were essentially equally effective in retrieving sample traces (see Lett, 1979). The important variable yielding near equivalence of performance may have been that all stimuli were in the auditory modality, which is apparently extremely flexible in its processing capability (Herman, 1980). Similar findings on processing flexibility have been reported for manipulation of information in the visual modality for capuchin monkeys (D'Amato, 1973; D'Amato \& Worsham, 1974).

An alternative theoretical interpretation of the delayed-matching data is that the postdelay comparison sounds have no retrieval function whatsoever. The auditory memory functions obtained in delayedmatching tasks may simply reflect the dolphin's ability to maintain, perhaps via some mechanism analogous to human rehearsal (Wagner, Rudy, \& Whitlow, 1973), encoded memory traces of the sample sounds. According to this view, comparison stimuli would serve simply to mark the end of the retention interval and indicate the available response loci.

If this particular explanation is valid, if the dolphin's working memory for sounds truly operates independently of the specific nature and modality of postdelay events, it should be possible to obtain comparable memory functions using the same sample sounds in conjunction with nonauditory comparison stimuli having different response requirements.

In the current study, the retrieval cues were removed from the auditory domain, but the to-be-remembered items remained different sounds and in fact were the same sounds used in the delayed-matching study discussed above. Visual-spatial cues-specifically, the different loci of underwater response paddles-were to be associated with different sounds. To demonstrate memory for the sound, the dolphin had to respond to the correct paddle after a delay. The major variable across the two experiments was the availability of overt orientations as mediating responses to bridge the delay interval. In Experiment 1, these responses were unavailable or at least difficult to develop, while in Experiment 2, they were potentially available and in fact did develop.

\section{GENERAL METHOD}

\section{Subject}

The subject was an adult female bottlenosed dolphin (Tursiops truncatus) named Kea Kiko ("Kea"), approximately $141 \mathrm{~kg}$ in weight and 10 or 11 years of age at the time the study was carried out. She was a highly test-sophisticated animal, the subject of many studies of auditory learning, memory, and sensory processes in the dolphin (Herman, 1975; Herman \& Arbeit, 1972, 1973; Herman \& Gordon, 1974; Thompson \& Herman, 1975, 1977; Yunker \& Herman, 1974; Herman \& Thompson, Note 1).

\section{Apparatus}

Kea was tested in her home tank-an outdoor, circular, concrete enclosure, $15.2 \mathrm{~m}$ in diameter, with a seawater depth of approximately $1.5 \mathrm{~m}$. Figure 1 is a schematic drawing of the experimental arrangement. Two pairs of vertically suspended ropes defined an underwater channel leading to a start paddle at $.6 \mathrm{~m}$ depth. The "listening area" was a favorable region of high sound intensity in which the dolphin listened to the projected sounds. The main sound projector (center speaker) was a Sumner and Mills $\mathbf{J} 9$ underwater transducer. The paddles, shown in Figure 1, were suspended from overhead beams and extended into the water. Lateral movements of a paddle triggered a microswitch. The two side speakers were University MM2PPS underwater transducers.

In the two experiments reported here, both pairs of paddles were simultaneously in the water only in Experiment 1. In Experiment 2, either the left-hand or right-hand pair was present within a given session.

\section{Sounds}

Two sounds, labeled " $A$ " and "B," were used as the to-beremembered pre-delay sounds in all procedures. Sound $A$ was a $4-\mathrm{kHz}$ sinusoidal constant frequency (CF) signal modulated at a $3-\mathrm{Hz}$ rate to $\pm 25 \%$ of the center frequency. Sound B was a $25-\mathrm{kHz}$

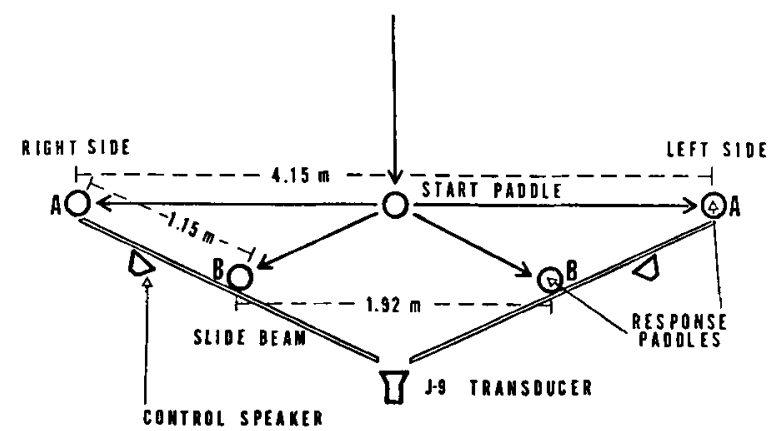

Figure 1. Schematic drawing of the apparatus used in Experiments 1 and 2. In Experiment 1, both paddle pairs were simultaneously in the water. In Experiment 2, either the left-hand or the right-hand paddle pair was present within a given session. In both Experiments 1 and 2, sample sounds $A$ and $B$ were presented from the center speaker. Postdelay "exit" sounds were presented from the appropriate side speaker. 
CF signal. A and B were the same sounds used as samples in the cited auditory delayed-matching study of Herman and Thompson (Note 1).

Two complex waveform sounds, with primary frequencies of 221 and $522 \mathrm{~Hz}$, were used to control the dolphin's entry into the listening area and to conditionally reinforce correct responses. They were presented from the center speaker. Two additional complex sounds, with primary frequencies of 300 and $650 \mathrm{~Hz}$, controlled the dolphin's choice of paddle pair (left or right). These sounds were presented from the respective side (control) speakers.

All sounds were generated by voltage-controlled oscillators (Wavetek 131A or 136; Eico 377), alone or in combination. The signals were calibrated before each testing session with a digital electronic counter (General Radio 1192) and oscilloscope (HewlettPackard 120B).

For sounds $\mathrm{A}$ and $\mathrm{B}$, the signal voltage that was applied to the center J9 speaker, via a Hewlett-Packard 467A power amplifier, was randomly either 6.4 or $7.1 \mathrm{~V}$ rms, producing mean in-water signal levels of 33 to $37 \mathrm{~dB}$ (re: $1 \mu \mathrm{bar}$ ) for $A$ and 46 to $50 \mathrm{~dB}$ for $\mathrm{B}$, at $1 \mathrm{~m}$ from the speaker. Signal levels in the listening area were measured in the manner described by Thompson and Herman (1975).

Signal durations, interstimulus intervals, and intertrial intervals were controlled by electronic timers. Sequencing of events within and between trials was controlled through solid-state programming logic.

\section{General Procedure}

Each discrete trial began with onset of a 10-sec limited-hold call tone $(221 \mathrm{~Hz})$ from the center speaker. In response, the dolphin entered the listening area through the rope channel, pressed the start paddle, and turned off the call tone. After a 4-sec pause, either sound A or B was projected from the center speaker for $2.5 \mathrm{sec}$. After the prescribed delay interval, which was progressively increased over sessions each time the animal met a predetermined performance criterion, the dolphin was cued to respond to either the left-hand or right-hand paddle pair by a continuous "exitsound" projected from one or the other of the two side speakers positioned between each paddle pair. If both pairs of paddles were present in the water, the exit sound removed the left-right spatial ambiguity and released the animal to make a response on a near or far paddle of the pair. If only one pair was present, spatial ambiguity was already resolved, and the exit sound acted only to release the dolphin to respond to one of the two paddles of the pair.

Pressing a paddle ended the exit sound and, if the response was correct, immediately produced playback of the sound heard prior to the delay (A or B). A .5-sec conditioned reinforcement sound was then projected from the center speaker, and a silver smelt (Osmerus sp., approximately $34 \mathrm{~g}$ in weight) was thrown into the tank as reward. After an intertrial interval (ITI) of 15 or $30 \mathrm{sec}$, the next trial began. The dolphin was rewarded for pressing the outer paddle of a pair following sound $A$ and for pressing the inner paddle of a pair following sound $B$. Responses to the incorrect paddle of a pair also ended the exit sound, but produced playback of the sound that had not been heard prior to the delay, which was followed immediately by the ITI.

Training. Training made use of prior findings that acquisition of spatial responses to nonspatial sounds by the dolphin is facilitated if a spatial localization cue is used initially and then gradually eliminated (Yunker \& Herman, 1974). Initially, only a single paddle pair was used during a training session. Use of the left and right paddle pairs was alternated over successive sessions. Two J9 speakers were positioned adjacent to the two response paddles of a pair, providing a spatial cue to indicate the correct paddle when sound A or sound B appeared on the appropriate speaker. Over successive sessions, this spatial cue was gradually eliminated by first moving the two speakers toward a center position between the paddle pair and then finally using only a single speaker midway between the pair.
The dolphin was trained next to accommodate to both sets of paddles in the water at the same time. During trials of correct re sponding to a given pair, the alternate pair was gradually placed in the water. The number of trials taken within a session to introduce the second paddle pair was progressively reduced until finally both pairs were in the water from the beginning. The two side speakers were then introduced. Now the exit-sound signaling the animal to leave the listening area and make a response to either the left-hand or right-hand paddle pair was projected from the appropriate left or right side speaker. Also, during this stage of training, the left-hand and right-hand J9 speakers were moved gradually toward a position midway between the two sets of paddles. Finally, the two J9 speakers were replaced by the single $\mathrm{J} 9$ center speaker.

Testing. During the testing phase, the delay between the end of sound $A$ or sound $B$ and onset of either the left- or right-hand exit sound was gradually increased across successive sessions. As in the auditory delayed-matching study (Herman \& Thompson, Note 1), two discrete delay values were tested in each session, the longer delay value in the previous session becoming the shorter delay value in the next session. For example, the delay pair 1 and $2 \mathrm{sec}$ was used in the first session, 2 and $3 \mathrm{sec}$ in the next session, 3 and $4 \mathrm{sec}$ in the next, and so on. Each delay value in a particular pair differed by $1 \mathrm{sec}$ through pair $9-10 \mathrm{sec}$, by $2 \mathrm{sec}$ from pair 10-12 through pair 16-18, by $4 \mathrm{sec}$ from pair $18-22$ through pair $30-34$, by $6 \mathrm{sec}$ from pair 34-40 through pair 52-58, and by $8 \mathrm{sec}$ for pair $58-66 \mathrm{sec}$, the final pair tested.

There were two daily 84-trial sessions separated by a 1 - to 2 -h break. Each session consisted of two parts separated by a 15- to 20-min break, and each part of a session was subdivided into an 18-trial warm-up block and a 24-trial test block. The order of the two ITI values ( 15 and $30 \mathrm{sec}$ ) was counterbalanced across successive sessions. Further details of the warm-up and test blocks are given in the descriptions of Experiments 1 and 2.

\section{EXPERIMENT 1}

Experiment 1, an indirect auditory delayed discrimination, tested for the retention of familiar sounds by the dolphin in a situation in which overt mediational cues, such as body orientation toward a response site, were not readily available. The left-hand and the right-hand pairs of paddles were in the water at the same time (Figure 1). The dolphin was rewarded for responding on the outer paddle of a pair after hearing sound $A$ from the centrally placed underwater speaker and on the inner paddle of a pair after hearing the alternative sound $\mathrm{B}$. The dolphin's memory for A or B was tested by progressively increasing the delay between the end of the sample sound and the opportunity to respond on a paddle. The dolphin was not cued as to which paddle pair, left or right, to choose from until after the retention interval had ended, a procedure that guarded against the use of overt body orientations toward any particular paddle during the delay interval.

\section{Procedure}

Warm-up block. The 18 warm-up trials included, successively: (1) four trials with either the two outer-A paddles or the two inner-B paddles in the water; (2) six trials with the addition of a third paddle (e.g., two B paddles and one A paddle or the reverse), with each of the three paddles tested for two consecutive trials in a systematic order; and (3) eight trials with all four paddles in position, with each paddle tested twice in a systematic order. Throughout the experiment, no errors were ever made during 
Parts 1 and 2, but errors might occur during Part 3, which was identical in format to the test phase that followed.

If the animal's performance in Part 3 was $75 \%$ correct $(6 / 8$ correct responses) or better, with no more than one error per paddle, the 24-trial test block was started immediately; otherwise, the warm-up procedure was reinstated at Part 2 . If the $75 \%$-correct criterion was not met within three successive attempts, the warmup was considered to have been failed, and the session was terminated. The delays used during warm-up were the same as those planned for the test phase; hence, a failure of warm-up was considered to be a failure to successfully bridge the delay under the test condition of Part 3.

Test block. Each 24-trial test block was balanced such that each side, paddle, and delay interval combination occurred once every eight trials in a quasi-random order determined from a large pool of such sequences.

Beginning with the 1- and 2-sec delay pair, successive sessions tested increasing delay pair values as described earlier. This procedure was continued until either the 18-trial warm-up criterion was failed during a session or the animal self-terminated a session. Self-terminations occurred only after error rates rose considerably, and were defined as a failure of Kea to respond to a call tone signaling the start of a new trial.

Failure to pass the warm-up criterion or self-termination of a session was considered to estimate the limit of delay for the test run, and a new replication, beginning again with the 1 - and 2-sec delay pair, was started immediately at the next session. A total of three replications were completed in this manner.

\section{Results and Discussion}

Training of Kea for these procedures took place over 47 sessions, encompassing 25 days and a total of 5,726 trials.

The mean percentage of correct responses at each tested delay value is shown by the dashed lines in Figure 2. The number of test trials comprising each data point ranged from 72 , at the 1 -sec delay, to 144 , except for the terminal 30-sec data point, which is based on only 24 test trials. The dolphin's performance was better than $90 \%$ correct through the 16-sec delay and then progressively decreased to a minimum of $79 \%$ correct responses at the $26-\mathrm{sec}$ delay. The dolphin failed to meet the warm-up criterion during the 30 and 34-sec delay pair session on the final replication, and she self-terminated sessions with the 22 - and 26-sec delay pair and 26- and 30-sec delay pair on the first and second replications, respectively. The data across replications were analyzed for possible systematic practice effects. No significant differences in performance levels between any pair of replications (1 vs. 2 , 1 vs. 3 , and 2 vs. 3) were revealed by the results of separate Wilcoxon matched-pairs tests (Siegel, 1956). In other words, there was no significant practice effect across replications.

Figure 2 also shows that Kea's performance level at the shortest, 1 - and 2-sec, delay values was actually somewhat below that obtained at the longer, 3- to 6-sec, delay values. This particular finding may simply reflect some continued acquisition of the conditional association between the sample sounds and the spatial responses, since the shortest delays were always tested first. A similar result was found in the first of three experiments in the delayed-matching study (Herman \& Thompson, Note 1), in which delay

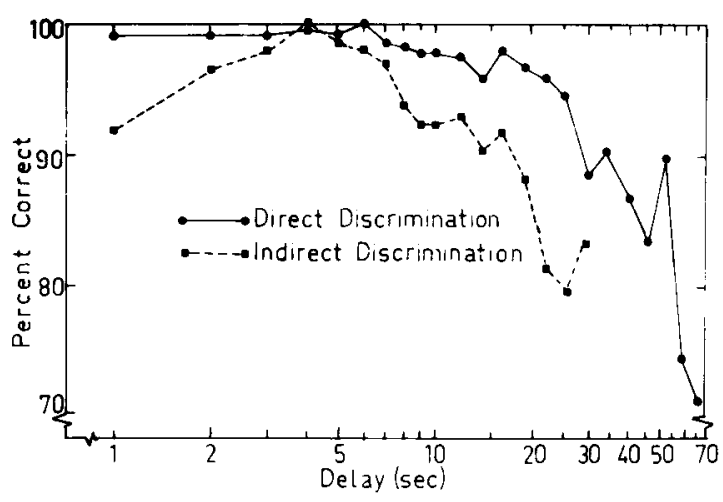

Figure 2. Mean percentage of correct responses at each delay tested for both the indirect (four-paddle, Experiment 1) and direct (two-paddle, Experiment 2) auditory delayed discriminations.

values were incremented in the same manner as the current study, but not in another delayed-matching task, in which the various delays occurred in a random, but balanced, presentation order (Herman \& Gordon, 1974).

A Wilcoxon matched-pairs test (Siegel, 1956) revealed no significant differences in the number of errors made following sounds A and B. There was also no significant difference in the effects of the 15and 30-sec ITIs on overall error rates. However, significantly more errors overall $(p<.005)$ were made by Kea to the left-hand paddle pair than to the righthand paddle pair. There was no apparent reason for this difference, and it may simply reflect a spatial bias manifested under conditions of response uncertainty.

To examine for proactive interference effects from the trial preceding the current one, the probability of an error was computed when the to-be-remembered sound was changed across two successive trials (i.e., trial digrams) and when it remained the same (see Table 1). As was found for delayed-matching tasks (Herman, 1975; Herman \& Thompson, Note 1), changing the sound across trials resulted in significantly more errors than when the same sound was presented again $\left(\chi^{2}=10.43, p<.005\right)$, suggesting a loss in the discrimination of relative recency of the sounds over time (cf. D'Amato, 1973; Herman, 1975). However, there must be more to it than that. In the delayed-matching tasks (Herman \& Thompson, Note 1), Kea's performance level remained above $90 \%$ correct for delays of up to $50 \mathrm{sec}$ and typically remained above $80 \%$ correct responses through the maximum 2- to 3-min delay limits at which the dolphin self-terminated test sessions. In the present delayed-discrimination task, Kea's performance was better than $90 \%$ correct only through 16 -sec delay values. Although she responded well above chance levels for the remaining delays, she either failed to meet the warm-up criterion or self-terminated sessions with delay values no greater than $30 \mathrm{sec}$. If the dolphin's performance on both the delayed-matching and delayed-discrimination tasks simply reflected a 
Table 1

Probability of an Error After Changing and After Not Changing the Test Sound Between Successive Trials (Digrams)

\begin{tabular}{|c|c|c|c|c|c|c|c|c|c|c|}
\hline \multirow{2}{*}{$\begin{array}{c}\text { Experi- } \\
\text { ment }\end{array}$} & \multirow{2}{*}{$\begin{array}{l}\text { Total } \\
\text { Trials }\end{array}$} & \multirow{2}{*}{$\begin{array}{l}\text { Trial } 1 \\
\text { Errors }\end{array}$} & \multirow{2}{*}{$\begin{array}{c}\text { Total } \\
\text { Digrams }\end{array}$} & \multicolumn{3}{|c|}{ Changed Test Sound } & \multicolumn{3}{|c|}{ Same Test Sound } & \multirow[b]{2}{*}{ PCD } \\
\hline & & & & Digrams & Errors & $\mathrm{PE}$ & Digrams & Errors & PE & \\
\hline 1 & 2,105 & 3 & 2,009 & 1,227 & 112 & $10.10^{*}$ & 782 & 40 & 5.39 & 61.08 \\
\hline 2 & 4,135 & 6 & 3,959 & 2,466 & 150 & $6.08 \dagger$ & 1,493 & 44 & 2.95 & 62.29 \\
\hline
\end{tabular}

Note-The total number of digrams in a set of trials is one less than the number of trials. Trial 1 error indicates an error on the first trial of each session. $P E=$ percent error; $P C D=$ percent changed digrams. $\quad{ }^{*} \chi^{2}(1)=10.43, p<.005 . \quad+\chi^{2}(1)=18.95, p<.005$.

task-independent ability to remember which of two sample sounds had occurred most recently in the face of proactive interference from prior trials, then we might reasonably have expected comparable delay effects across tasks. Given these discrepancies in performance, it seemed imperative to reduce the task demands of Experiment 1 and provide a simple cuing situation that was more analogous to delayedmatching procedures.

\section{EXPERIMENT 2}

This experiment examined whether performance might be improved upon, possibly to levels comparable to those obtained in delayed-matching studies, if a more direct form of the auditory delayeddiscrimination task was used. To simplify matters, only one paddle pair, left or right, was used during an experimental session, the other pair being withdrawn from the water. This procedure made the task analogous to the conventional delayed-matching procedure in that only two postdelay response choices were available. Additionally, the procedure permitted the dolphin to potentially "bridge" the delay interval following sound A or B by orienting toward the correct paddle or by using other overt mediational cues (Fletcher, 1965). Direct delayed discriminations have been studied in the pigeon (Smith, 1967). Monkeys have also been trained to make a spatial response conditional upon an auditory discriminative stimulus (Dewson \& Cowey, 1969), but delay effects were not examined.

\section{Procedure}

Only one paddle pair, left-hand or right-hand, was used during a session, the other pair being withdrawn from the water. Except for minor changes in the warm-up criteria, the testing procedures were the same as in the indirect delayed-discrimination task. The 16 warm-up trials included: (1) four trials with a single paddle, either A or B, in the water; (2) four trials with only the alternate paddle in the water; and (3) eight trials with both paddles in the water, as in the succeeding test phase. Each paddle was tested twice in a row for two consecutive trials. As was the case in Experiment 1 , errors occurred only at this last stage. If the animal's performance in Part 3 was $75 \%$ correct (6/8 correct trials) or better, with no more than one error per paddle, the 24-trial test block was started immediately. Otherwise, the warm-up procedure was reinstated by withdrawing the paddle to which the animal was incorrectly responding. A session was terminated if the animal failed to meet the warm-up criterion after three successive attempts.

During the test phase, occurrence of sounds $\mathrm{A}$ and $\mathrm{B}$, together with the two delay intervals of a session, was quasi-randomly balanced across the 24 trials. The specific paddle pair used, either the left-hand or the right-hand pair, and the ITI were counterbalanced across successive sessions. Testing proceeded exactly as in Experiment 1, replications beginning with the 1- and 2-sec delay pair, and successive sessions testing successively longer pairs of delays. This procedure was continued until the 16-trial warm-up criterion was failed or the animal self-terminated a session. At this point, a new replication, beginning again at 1- and 2-sec delays, was initiated at the next session. A total of five replications were completed in this manner.

\section{Results and Discussion}

The mean percentage of correct responses at each delay tested is shown by the solid line in Figure 2. The number of test trials comprising each data point ranged from 108 , at $1-\mathrm{sec}$ delay, to 240 , except for the 58- and 66-sec delays, which were based on only 66 and 21 trials, respectively. Overall, the animal's performance remained at about $95 \%$ correct or better through 26-sec delays. Beyond the 30-sec delay interval, the limit of performance reached in Experiment 1 , Kea's performance in this experiment gradually and irregularly decreased to $75 \%$ correct responses at the 58-sec delay interval and to $71 \%$ correct at the 66-sec delay. Kea failed to meet the warm-up criterion during the 34- and 40-sec delay session in the first replication and self-terminated the sessions with the 52-58- and 58-66-sec delay pairs in the first and final three replications, respectively.

The results of a Wilcoxon matched-pairs test revealed that the overall performance differences between the final replication of Experiment 1 and the first replication of the present experiment were not significant. The Wilcoxon test was also used to compare performances across all possible replication pairs in the present experiment. Overall performances on the first and second replications differed significantly $(p<.05)$, as did the differences between the second and third replications $(p<.05)$. However, differences in overall performance levels between any pair of the final three of the five replications were not significant.

During the longer delays (exceeding $30 \mathrm{sec}$ ) of all replications except the first, we observed that the dolphin typically moved forward in the listening area on $70 \%$ to $100 \%$ of those trials on which she responded to the outer paddle (associated with sound A), but on no more than $25 \%$ of those trials on which she responded to the inner paddle (associated with sound B). The percentage occurrence of the overt response at each delay value is shown in Figure 3, which reveals 
that the forward movement response developed as the delay interval lengthened beyond $4 \mathrm{sec}$ and greatly strengthened at delays greater than $12 \mathrm{sec}$.

As in Experiment 1, Wilcoxon matched-pairs tests revealed no significant differences in the number of errors made in sessions with the 15- and 30-sec ITI. Nor was there any significant difference in the number of errors made following A vs. B test sounds or to the left-hand vs. right-hand paddle pairs. The probability of an error, however, was again significantly greater following a between-trial change in the to-be-remembered sound than when the sample sound remained the same (Table $1, \chi^{2}=18.95$, $\mathrm{p}<.005$ ).

The delay limits reached by Kea in this experiment were about twice as long as those reached in Experiment 1 . The increased delay limit cannot be dismissed as a simple practice effect resulting from the order in which the two experiments were run. There were no significant differences in either delay limit or overall performance between the first replication of this experiment and the final replication of Experiment 1. The significant improvement in this experiment between the first and subsequent replications was correlated with the appearance of overt postural responses, particularly at the longer delays, that reliably predicted paddle responses. Finally, there were no significant differences in either delay limit or overall performances across the final three replications. Our observation of response-specific overt responses is consistent with reports from delayed-response studies that a variety of species may adopt postural cues to bridge retention intervals when given the opportunity, even though such responses are not a necessary condition for good performance (Fletcher, 1965).

Despite the facilitation of Kea's performance, which apparently resulted from the use of overt mediational responses, the 1-min delay limit that was reached still fell considerably short of the 2- to 3-min delay limits obtained in auditory delayed-matching studies with the dolphin Kea (Herman \& Gordon, 1974; Herman \& Thompson, Note 1).

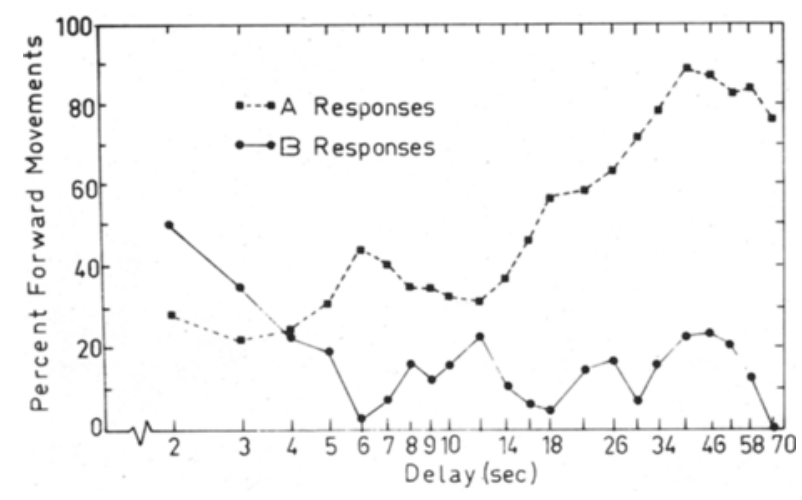

Figure 3. Percent occurrence of forward movements observed during the delay interval in the direct delayed discrimination (Experiment 2).

\section{GENERAL DISCUSSION}

In the current study, a conditional relationship was learned by a dolphin between two sounds and the loci for responses to those sounds when the loci were other than the site of the sounds themselves. Memory for the sounds was then tested by requiring that the spatial responses to a sound be withheld until a prescribed delay interval had elapsed. The findings indicate that retention of sounds on the order of 30 to $60 \mathrm{sec}$ was possible within this memory paradigm. The greater retention time was obtained in a condition permitting development of overt postural cues to the correct locus during the delay, and indeed such overt postures appear to have developed spontaneously as delays lengthened. It is clear that the association itself was well learned, and it is highly unlikely, therefore, that performance decrements at the longer delays in either experiment are attributable to a loss of reference memory (Honig, 1978).

It is noteworthy, however, that even with the aid of postural mediational responses as memory aids, the delay intervals spanned by the dolphin fell short, by approximately $1 \mathrm{~min}$, of those consistently attained in delayed-matching studies in which postdelay comparison cues (retrieval cues), like the to-be-remembered predelay items, were auditory (Herman \& Thompson, Note 1). In the Herman and Thompson study, it did not matter whether the retrieval cues were directly or only conditionally related to the to-be-remembered sample sounds: Performance was at the same high level in either case. The important point of comparison between the present study and that of Herman and Thompson is that the to-be-remembered sounds and the dolphin subject were exactly the same; only the postdelay comparison retrieval cue varied from intramodality in the Herman and Thompson study to intermodality in this study.

If performance levels over time simply indexed a failure to faithfully maintain stored traces of the sample sounds in the face of interference from sounds stored on previous trials, then the type of response or task, delayed matching or delayed discrimination, ought not to matter. But comparisons across studies indicate that it does indeed matter. Why the performance differences should be obtained for the same sounds under the two conditions is not readily apparent, but obviously the memory functions cannot depend on the sample alone. They must be determined in part either by retrieval or discriminative processes at the end of the retention interval, or else in the way information is encoded and stored in memory.

If we assume that the paddles used in the present study function as retrieval cues, then the data imply that visual-spatial stimulus attributes, perhaps because of the cross-modal associations involved, are not as effective at retrieving sample sounds as were the comparison sounds used in the delayed-matching studies. Perhaps the presence of the paddles contin- 
uously throughout the delay generates increasing amounts of interference from competing memories. In contrast, the problem of retrieval-cue-generated interference in standard delayed-matching tasks does not arise because the retrieval cues are presented only at the end of the retention interval.

An alternative theoretical possibility is that the dolphin does not encode and store traces of the sample sound, but rather, encodes and stores traces of anticipatory responses elicited by sample sound presentation. According to this type of response-oriented encoding interpretation, it is the response tendency to a specific postdelay event that must be maintained over time to be released or retrieved and then executed at the end of the retention interval (Blough, 1959; Cumming, Berryman, \& Cohen, 1965; Honig, 1978; Lawrence, 1963). Proactive interference effects from prior trials might reflect the increased difficulty of remembering-not which sample occurred most recently, but rather, which response option was most recently tagged as the locus for reward. Reducing the ambiguity of response location across experiments led to increased memory performance. This finding, correlated with the development of spatial overt mediational behavior, is consistent with an anticipatory response-encoding interpretation.

If the response-encoding interpretation has validity, then remembering the attributes of a nonauditory response locus is more difficult for the dolphin than remembering the attributes of an auditory response locus, at least in the paradigms tested in this study and in the auditory delayed-matching studies. Perhaps response tendencies to nonauditory events are especially prone to interference, either from the animal's own movements during the retention interval or because of competing response tendencies elicited by other paddles in the available and observable set of paddles.

Regardless of the specific nature of the underlying processes, the task differences force the conclusion that auditory working memory in the dolphin cannot depend upon memory for sample attributes alone. Some evidence for the comparative generality of this conclusion comes from results of recent studies of pigeon working memory using procedures other than delayed matching (Honig, 1978). Theories of animal working memory based primarily, if not solely, on the presumed fate of sample traces are apparently somewhat more limited in their utility than we previously might have assumed.

\section{REFERENCE NOTE}

1. Herman, L. M., \& Thompson, R. K. R. Encoding and retrieval processes in auditory short-term memory in the bottlenosed dolphin. Manuscript submitted for publication, 1980.
Cumming, W. W., Berryman, R., \& Cohen, L. R. Acquisition and transfer of zero-delay matching. Psychological Reports, $1965,17,435-445$.

D'Aмато, M. R. Delayed matching and short-term memory in monkeys. In G. H. Bower (Ed.), The psychology of learning and motivation: Advances in research and theory (Vol. 7). New York: Academic Press, 1973.

D'Aмато, M. R., \& Worsham, R. W. Retrieval cues and shortterm memory in capuchin monkeys. Journal of Comparative and Physiological Psychology, 1974, 86, 274-282.

Dewson, J. H., III, \& Cowey, A. Discrimination of auditory sequences by monkeys. Nature, 1969, 222, 695-697.

Fletcher, H. J. The delayed response problem. In A. M. Schrier, H. F. Harlow, \& F. Stolinitz (Eds.), Behavior of nonhuman primates (Vol. 1). New York: Academic Press, 1965.

Herman, L. M. Interference and auditory short-term memory in the bottlenosed dolphin. Animal Learning \& Behavior, 1975, 3, 43-48.

Herman, L. M. Cognitive characteristics of dolphins. In L. M. Herman (Ed.), Behavior of the delphinids. New York: WileyInterscience, 1980.

Herman, L. M., \& Arbeit, W. R. Frequency difference limens in the bottlenose dolphin: 1-70 kHz. Journal of Auditory Research, 1972, 12, 109-120.

Herman, L. M., \& Arbeit, W. R. Stimulus control and auditory discrimination learning sets in the bottlenose dolphin. Journal of the Experimental Analysis of Behavior, 1973, 19, 379-394.

Herman, L. M., \& Gordon, J. A. Auditory delayed matching in the bottlenose dolphin. Journal of the Experimental Analysis of Behavior, 1974, 21, 19-26.

HoniG, W. K. Studies of working memory in the pigeon. In S. H. Hulse, H. Fowler, \& W. K. Honig (Eds.), Cognitive processes in animal behavior. Hillsdale, N.J: Erlbaum, 1978.

KRUGER, L. Specialized features of the cetacean brain. In K. N. Norris (Ed.), Whales, dolphins, and porpoises. Berkeley: University of California Press, 1966.

Lawrence, D. H. The nature of a stimulus: Some relationships between learning and perception. In S. Koch (Ed.), Psychology: A study of a science (Vol. 5). New York: McGraw-Hill, 1963.

LETT, B. T. Long delay learning: Implications for learning and memory theory. In N. S. Sutherland (Ed.), Tutorial essays in experimental psychology (Vol. 2). Hillsdale, N.J: Erlbaum, 1979.

SIEGEL, S. Nonparametric statistics for the behavioral sciences. New York: McGraw-Hill, 1956.

Sмгтн, L. Delayed discrimination and delayed matching in pigeons. Journal of the Experimental Analysis of Behavior, 1967, 10, 529-533.

Spear, N. E. Forgetting as retrieval failure. In W. K. Honig \& P. H. R. James (Eds.), Animal memory. New York: Academic Press, 1971.

SPEAR, N. E. The processing of memories: Forgetting and retention. Hillsdale, N.J: Erlbaum, 1978.

Thompson, R. K. R., \& Herman, L. M. Underwater frequency discrimination in the bottlenosed dolphin $(1-140 \mathrm{kHz})$ and the human (1-8 kHz). Journal of the Acoustical Society of America, $1975,57,943-948$.

Thompson, R. K. R., \& Herman, L. M. Memory for lists of sounds by the bottlenosed dolphin: Convergence of memory processes with humans? Science, 1977, 195, 501-503.

Wagner, A. R., Rudy, J. W., \& Whitlow, J. W. Rehearsal in animal conditioning. Journal of Experimental Psychology, $1973,97,407-426$.

Yunker, M. P., \& Herman, L. M. Discrimination of auditory temporal differences by the bottlenose dolphin and by the human. Journal of the Acoustical Society of America, 1974, 56, 1870-1875.

\section{REFERENCES}

Blough, D. S. Delayed matching in the pigeon. Journal of the Experimental A nalysis of Behavior, 1959, 2, 151-160.
(Received for publication September 4, 1979; revision accepted August 12,1980 .) 